\title{
HELICAL FIELD LINES IN AN ERUPTING FILAMENT
}

JOAN VORPAHL

Dept. of Physics and Astronomy, Sacramento City College, Sacramento, Calif., U.S.A.

\begin{abstract}
An example of an erupting filament that exhibited helical structure during a flare on 1971, September 17, was discussed. This sort of event is not rare. Examples of rotational effects in flares, prominences and surges have been reported by others such as Öhman (1968). Accompanied by type II radio emission and the visible flow of $\mathrm{H} \alpha$ gas across $\frac{1}{2}$ the Sun's surface, the flare was very interesting in itself. However, here the emphasis is on possible ways in which the observed twist might develop. The latter is important because it yields information about:(1) the state of the subphotospheric field itself, and (2) the possibility of storing flare energy in the twisted filaments.

Possible reasons for observing twisted field lines include:

(1) Photospheric motion resulting in twisted flux tubes is attractive because various polarities or rotation of sunspots;
\end{abstract}

(2) minor field reconnection occurs in which new flux interacts with fields already present and produces intertwining filaments;

(3) flux tubes come up twisted and merely expand into the chromosphere.

Considering each of these in turn for the September event:

(1) Photospheric motion resulting in twisted flux tubes is attractive because various authors have shown that field lines can be twisted by a rotating sunspot (Barns and Sturrock, 1971) or by opposite polarity regions slipping past one another (Nakagawa and Tanaka, 1973). Two ways of determining shearing motion include: (a) sunspot motion or (b) high spatial and temporal resolution magnetograms. In the case of the September flare, no high resolution magnetograms exist. However, the twist did not seem to be due to shearing because: (a) no obvious motion (relative to a nearby larger active region) occurred in the bright plage region that constituted the flare site; (b) no surrounding $\mathrm{H} \alpha$ features such as fibril structures, appeared to move; and (c) the appearance of the twist was quite abrupt, i.e., it became obvious within a period of $1-2 \mathrm{~h}$, on the day preceding the flare. Since opposite ends of some fibrils were displaced about 20000 to $25000 \mathrm{~km}$, the necessary velocity of photospheric motions in explaining the helical filaments would be prohibitively high.

(2) Minor field reconnections could also result in filament twist. In the case under consideration, filaments encircling the bright plage did undergo oscillations, appearing first lighter then darker, several times on the day before the flare. On the other hand, it is not obvious as to why field reconnections would occur since they would result in a twisted field - and presumably a higher energy state.

(3) Due to subphotospheric activity, flux tubes might come up twisted. This is consistent with the September flare in that the twist became obvious over a short period of 1-2 h. Furthermore, no obvious photospheric or chromospheric motions were observed. On the other hand, no twist is noticeable in fibrils that make up arched 
filaments in a newly emerging magnetic region; however, this might be due to the inadequacy of current telescopes to resolve fine structure in tightly wound flux ropes.

In summary, helical structure in the September 1971 filament can be explained most easily by the fact that flux tubes came up twisted. However, since magnetograms of high quality resolution did not exist, photospheric motion that resulted in twisted filaments cannot be excluded.

\section{References}

Barns, C. and Sturrock, P.: 1971, Astrophys. J. 174, 659.

Nakayawa, Y. and Tanaka, K.: 1973, High Altitude Observatory, preprint.

Ohman, Y. (ed.): Mass Motion in Solar Flares and Related Phenomena, Wiley Interscience Division, New York.

\section{DISCUSSION}

Giovanelli: The question of twisted field lines is connected with the question that has been worrying me for quite some time. Many years ago Bray and Loughhead observed some small sunspots that appeared very suddenly and migrated over to join with another sunspot thereby increasing its size. These observations were drawn to my attention when I saw Harold Zirin's observations this morning of the way flux regions emerged. How is it that flux comes up and gets gathered into sunspots? Is it because it comes up twisted or perhaps shredded apart in the photosphere but then when it spreads out these twists don't let it stay apart? It's a question I have to ask and I'd love to hear what somebody else has to say about it.

Meyer: This afternoon I am going to talk a little about the problem how sunspots come about and decay. It seems that the twist is not an essential feature in our model.

Newkirk: Can you make an estimate of how much energy is involved in the twist?

Vorpahl: The field lines in the twisted regions are about 20000 to $30000 \mathrm{~km}$ long and have about two or three twists in them. I have not tried to estimate the energy. 